\title{
Author Correction: Nanoscale control of competing interactions and geometrical frustration in a dipolar trident lattice
}

\author{
Alan Farhan (1) ${ }^{1}$, Charlotte F. Petersen ${ }^{2}$, Scott Dhuey ${ }^{3}$, Luca Anghinolfi ${ }^{4}$, Qi Hang Qin ${ }^{5}$, Michael Saccone ${ }^{6}$, \\ Sven Velten ${ }^{7,8}$, Clemens Wuth ${ }^{9,10}$, Sebastian Gliga ${ }^{11}$, Paula Mellado ${ }^{12}$, Mikko J. Alava ${ }^{2}$, Andreas Scholl ${ }^{1}$ \& \\ Sebastiaan van Dijken ${ }^{5}$
}

Nature Communications 8:995 10.1038/s41467-017-01238-4; Article published online: 17 October 2017

The original version of this article contained an error in the legend to Figure 4 . The yellow scale bar should have been defined as ' 600 nm', not ' $600 \mu \mathrm{m}$ '. This has now been corrected in both the PDF and HTML versions of the article.

Published online: 12 December 2017

\begin{abstract}
ceproduction in any medium or format, as long as you give appropriate credit to the original author(s) and the source, provide a link to the Creative Commons license, and indicate if changes were made. The images or other third party material in this article are included in the article's Creative Commons license, unless indicated otherwise in a credit line to the material. If material is not included in the article's Creative Commons license and your intended use is not permitted by statutory regulation or exceeds the permitted use, you will need to obtain permission directly from the copyright holder. To view a copy of this license, visit http://creativecommons.org/licenses/by/4.0/.
\end{abstract}

(C) The Author(s) 2017

\footnotetext{
${ }^{1}$ Advanced Light Source, Lawrence Berkeley National Laboratory (LBNL), 1 Cyclotron Road, Berkeley, CA 94720, USA. ${ }^{2}$ COMP Centre of Excellence, Department of Applied Physics, Aalto University, P.O. Box 11100, Espoo Fl-00076 Aalto, Finland. ${ }^{3}$ Molecular Foundry, Lawrence Berkeley National Laboratory (LBNL), 1 Cyclotron Road, Berkeley, CA 94720, USA. ${ }^{4}$ Dipartimento di Fisica, Università di Genova, via Dodecaneso 33, I-16146 Genova, Italy. ${ }^{5}$ NanoSpin, Department of Applied Physics, Aalto University School of Science, P.O. Box 15100, FI-00076 Aalto, Finland. ${ }^{6}$ Department of Physics, University of California, Santa Cruz, CA 95064, USA. ${ }^{7}$ Materials Sciences Division, Lawrence Berkeley National Laboratory, 1 Cyclotron Road, Berkeley, CA 94720, USA. ${ }^{8}$ Institut für Nanostruktur- und Festkörperphysik, Universität Hamburg, Jungiusstrasse 11, 20355 Hamburg, Germany. ${ }^{9}$ Center for X-ray Optics, Lawrence Berkeley National Laboratory, 1 Cyclotron Road, Berkeley, CA 94720, USA. ${ }^{10}$ Daegu Gyeongbuk Institute of Science and Technology (DGIST), 50-1 Sang-ri, Hyeonpung-myeon, Dalseong-gun, Daegu 42988, Republic of Korea. ${ }^{11}$ SUPA, School of Physics and Astronomy, University of Glasgow, Glasgow G12 $8 Q Q$, UK. ${ }^{12}$ School of Engineering and Sciences, Adolfo Ibáñez University, Diagonal Las Torres, 2640 Peñalolén, Santiago, Chile. Correspondence and requests for materials should be addressed to A.F. (email: alan.farhan@gmx.net)
} 